\title{
Exact expression for the diffusion propagator in a family of time-dependent anharmonic potentials
}

\author{
J. A. Giampaoli* ${ }^{*}$ D. E. Strier ${ }^{\dagger}$, C. Batista, German Drazer ${ }^{\ddagger}$ and H. S. Wio ${ }^{\S}$ \\ Comisión Nacional de Energía Atómica, Centro Atómico Bariloche and Instituto Balseiro (CNEA \\ and UNC); 8400-San Carlos de Bariloche, Argentina.
}

(July 8, 2021)

\begin{abstract}
We have obtained the exact expression of the diffusion propagator in the timedependent anharmonic potential $V(x, t)=\frac{1}{2} a(t) x^{2}+b \ln x$. The underlying Euclidean metric of the problem allows us to obtain analytical solutions for a whole family of the elastic parameter $a(t)$, exploiting the relation between the path integral representation of the short time propagator and the modified Bessel functions. We have also analyzed the conditions for the appearance of a non-zero flow of particles through the infinite barrier located at the origin
\end{abstract}

\footnotetext{
*Present address: Center for Industrial Research, FUDETEC; Av.Córdoba 320, 1er. piso; 1054Buenos Aires; Argentina

${ }^{\dagger}$ Electronic address:strier@df.uba.ar, Present address: Departamento de Física; Facultad de Ciencias Exactas; Universidad de Buenos Aires; Pabellón I, Ciudad Universitaria, 1428-Buenos Aires; Argentina

${ }_{\ddagger}^{\ddagger}$ Electronic address:gdrazer@tron.fi.uba.ar, Permanent address: Grupo de Medios Porosos, Facultad de Ingeniería, Universidad de Buenos Aires, Paseo Colón 850. CP 1063, Argentina

§E-mail: wio@cab.cnea.gov.ar;

http://www.cab.cnea.gov.ar/CAB/invbasica/FisEstad/estadis.htm
} 
$(b<0)$.

\section{INTRODUCTION}

The mathematical theory of stochastic processes has proven to be not only a useful but also a necessary tool when studying physical, chemical and biological systems under the effect of fluctuations [1]. Recent theoretical and experimental studies have shown that there are many situations where fluctuations plays an essential role leading to new phenomena induced by the presence of noise. A few examples of such situations are: some problems related with self-organization and dissipative structures [2, 3], noise induced transitions [4], noise induced phase transitions [5], noise sustained patterns [6], stochastic resonance in zero-dimensional and in spatially extended systems [7,8].

An almost natural way to describe (Markovian) stochastic processes corresponds to an approach introduced by Wiener based on a sum over trajectories [9], anticipating by two decades Feynman's work on path integrals [10]. This approach was later applied by Onsager and Machlup to some Markovian nonequilibrium processes [11]. However, even some nonMarkov processes can be also described within this framework [12]. In spite of the historical fact, path and functional integration methods have been largely studied and applied within the quantum mechanical realm, while its study and application in stochastic processes is scarce [13]. However, it is worth here to remark that, following earlier results [14, path and functional integral methods have been widely applied in the theory of dynamic critical phenomena, both at equilibrium and nonequilibrium phase transitions [15].

Among others, one of the problems that has not received much attention within the path integral description of stochastic processes is the application of space-time transformations, while this kind of transformations have been largely used within the realm of quantum

mechanics [16]. Among the few studies in this regard (see Ref. [17]) a recent one refers to a transformation relating the diffusion propagator in a time-dependent harmonic oscillator 
with the propagator for the case of free diffusion, including a whole family of possible analytical solutions [18]. Also, a formal adaptation of Duru-Kleinert-like transformations to the stochastic case, overcoming the main disadvantage of the direct application of such transformations - namely that Duru-Kleinert transformations do not link different Markov processes- was introduced in Ref. [19]. However, it merits to stand out the treatment of transformations between "different" Wiener processes done in Friendlin's book (indicated with (j) in Ref. [13), even though they are not the exact equivalent to the Duru-Kleinert transformations.

In this paper we present the exact solution for the diffusion propagator in a timedependent anharmonic oscillator $V(x, t)=\frac{1}{2} a(t) x^{2}+b \ln x$. This particular choice of the potential can be useful to model the behavior of several physical and biological systems. Among them, the study of neuron models (e.g. integrate and fire models [7]), stochastic resonance in monostable nonlinear oscillators [20] and its possible application to spatially extended systems [8]. Also, we can consider that the logarithmic term, whithin the path integral scheme (or as a Boltzmann like weight) mimics a prefactor corresponding to an effective energy barrier. It is clear that the possibility of having exact expressions of the stochastic propagator in a non-symmetrical potential can be of interest. In fact, in many of the above mentioned applications (specially on neuron models) the potential studied in this work would represent a more realistic approximation to the real behavior of the system under study. In other problems such us brownian motors, this asymmetry is not just an improvement but an unavoidable ingredient of the model.

The approach used in this work has been inspired by the solution presented in Ref. [21], corresponding to the exact quantum mechanical propagator in a time-dependent harmonic potential plus a singular perturbation. In the present case, the fact that the metric of the underlying space is Euclidean, allows us to obtain the exact analytical expression of the diffusion propagator for a whole family of functional forms of the time-dependent elastic parameter.

In the next section we introduce the model we are going to study and show the proce- 
dure to be followed in order to obtain the exact form of the propagator. We also discuss the presence of noise induced flow of particles through the infinite barrier located at the origin, provided that the noise amplitude is large enough for the particles to overcome the deterministic drift. In section $[1]$ we show how to obtain a family of analytical solutions. In the last section we make a final discussion and comment on the possible applications of the present results.

\section{THE DIFFUSION PROPAGATOR}

In this section we will follow, and adequately adapt, the results of the paper of Khandekar and Lawande [21]. Our starting point is to consider the following Langevin equation

$$
\dot{x}=h(x, t)+\xi(t),
$$

where $\xi(t)$ is an additive Gaussian white noise [四]. That is it fulfills the conditions $\langle\xi(t)\rangle=0$ and $\left\langle\xi(t) \xi\left(t^{\prime}\right)\right\rangle=2 D \delta\left(t-t^{\prime}\right)$. This equation describes the overdamped motion of a particle in a time-dependent potential. In this work, we will consider the force term $h(x, t)=$ $-a(t) x-b / x$ which, through the relation $h(x, t)=-\partial V / \partial x$, corresponds to the following potential

$$
V(x, t)=\frac{1}{2} a(t) x^{2}+b \ln x .
$$

This potential is defined for $x>0$ and, whenever $b<0$ and the elastic parameter $a(t)$ is positive, it corresponds to an anharmonic monostable system composed by a time-dependent harmonic oscillator plus a logarithmic term which is singular at the origin.

As it will be shown later, even in this monostable situation, the noise could be able to induce a flow of particles through the infinite barrier located at the origin, overcoming the deterministic drift whenever $D>-2 b$ holds. In fact, the meaningful condition related to the conservation of particles inside the system (zero flux at $x=0$ ) is $D<-2 b$.

In this work we will relax the monostability condition, allowing for the time-dependent 
elastic parameter to take negative values. We will show that in this extended situation an asymptotic probability distribution can be reached whenever the elastic term satisfies,

$$
\lim _{t_{0} \rightarrow-\infty} \int_{t_{0}}^{t} a(s) d s=\infty \quad \forall t
$$

We will say that in this case the potential is strongly attractive.

The path integral representation of $P\left(x_{b}, t_{b} \mid x_{a}, t_{a}\right)$, that is the transition probability associated with this Langevin equation, is given by (see for instance the book indicated as "i" in [13])

$$
P\left(x_{b}, t_{b} \mid x_{a}, t_{a}\right)=\int_{x\left(t_{a}\right)=x_{a}}^{x\left(t_{b}\right)=x_{b}} \mathcal{D}[x(t)] \exp \left[-\int_{t_{a}}^{t_{b}} L(x(\tau), \dot{x}(\tau), \tau) d \tau\right] .
$$

Here the stochastic Lagrangian or Onsager-Machlup [11] functional is given, in a midpoint discretization, by

$$
L(x, \dot{x}, t)=\frac{1}{2 D}[\dot{x}-h(x, t)]^{2}+\frac{1}{2} \frac{\partial h(x, t)}{\partial x} .
$$

Replacing the actual form of $h(x, t)$ the previous expression can be expanded to yield

$$
L=L_{0}+\frac{d \Phi}{d t}
$$

where $\Phi$ corresponds to

$$
\Phi(t)=\left[\frac{b}{D}-\frac{1}{2}\right] \int_{t_{0}}^{t} a(\tau) d \tau+\frac{b}{D} \ln x+\frac{a(t) x^{2}}{2 D}
$$

with arbitrary $t_{0}$, and

$$
L_{0} \equiv \frac{1}{2 D}\left[\dot{x}^{2}+\left(a(t)^{2}-\dot{a}(t)\right) x^{2}+(b+D) \frac{b}{x^{2}}\right] .
$$

Hence the path integral in Eq. (4) adopts the form

$$
P\left(x_{b}, t_{b} \mid x_{a}, t_{a}\right)=e^{-\left[\Phi\left(t_{b}\right)-\Phi\left(t_{a}\right)\right]} K\left(x_{b}, t_{b} \mid x_{a}, t_{a}\right),
$$

with

$$
K\left(x_{b}, t_{b} \mid x_{a}, t_{a}\right)=\int_{x_{a}}^{x_{b}} \mathcal{D}[x(t)] \exp \left[-\frac{1}{2 D} \int_{t_{a}}^{t_{b}}\left(\dot{x}^{2}+\omega(\tau) x^{2}+(b+D) \frac{b}{x^{2}}\right) d \tau\right]
$$


and $\omega(t)=a(t)^{2}-\dot{a}(t)$. As usual, the path integral in Eq. (10) is defined in a discretized form by

$$
K\left(x_{b}, t_{b} \mid x_{a}, t_{a}\right)=\lim _{N \rightarrow \infty} A_{N} \int \ldots \int \exp \left(-\sum_{j=1}^{N} S_{j}\left(x_{j}, x_{j-1}\right)\right) \prod_{j=1}^{N-1} d x_{j}
$$

with $N \varepsilon=t_{a}-t_{b}, t_{j}=t_{a}+j \varepsilon, x_{0}=x_{a} ; x_{N}=x_{b} ; A_{N}=[2 \pi D \varepsilon]^{-\frac{N}{2}} ;$ and

$$
S_{j}=S_{j}\left(x_{j}, x_{j-1}\right)=\varepsilon L_{o}\left(x_{j}, x_{j-1}\right)=\frac{1}{2 D}\left[\frac{x_{j}^{2}+x_{j-1}^{2}}{\varepsilon}+\varepsilon \omega_{j} x^{2}\right]-\left[\frac{x_{j} x_{j-1}}{D \varepsilon}+\frac{D\left(\theta^{2}-\frac{1}{4}\right) \varepsilon}{2 x_{j} x_{j-1}}\right] .
$$

Here

$$
\theta=\theta(b)=\frac{1}{2} \sqrt{1+\frac{4 b(b+D)}{D^{2}}}=\left|\frac{1}{2}+\frac{b}{D}\right|
$$

Up to first order in $\varepsilon$ (exploiting that $\varepsilon \ll 1$ ) we can use the following asymptotic form of the modified Bessel function

$$
\exp \left(\frac{u}{\varepsilon}-\frac{1}{2}\left(\theta(b)^{2}-\frac{1}{4}\right) \frac{\varepsilon}{u}+O\left(\varepsilon^{2}\right)\right) \approx \sqrt{\frac{2 \pi u}{\varepsilon}} I_{\theta(b)}\left(\frac{u}{\varepsilon}\right)
$$

Using the last expression with $u=x_{j} x_{j-1} / D$ the propagator of Eq.(11) may be cast to the following form

$$
\begin{aligned}
K\left(x_{b}, t_{b} \mid x_{a}, t_{a}\right)= & \lim _{N \rightarrow \infty}\left(\frac{1}{2 \pi D \varepsilon}\right)^{1 / 2} \int \ldots \int \prod_{j=1}^{N-1} d x_{j} \\
& \times \prod_{j=1}^{N} \exp \left[-\frac{1}{2 D \varepsilon}\left(x_{j}^{2}+x_{j-1}^{2}+\varepsilon^{2} \omega_{j} x_{j}^{2}\right)\right]\left(\frac{2 \pi x_{j} x_{j-1}}{D \varepsilon}\right)^{1 / 2} I_{\theta}\left(\frac{x_{j} x_{j-1}}{D \varepsilon}\right) .
\end{aligned}
$$

The last expression can be rewritten as

$$
K\left(x_{b}, t_{b} \mid x_{a}, t_{a}\right)=\exp \left[\frac{-\beta}{2}\left(x_{a}^{2}+x_{b}^{2}\right)\right] \lim _{N \rightarrow \infty} \beta^{N} \int \ldots \int \prod_{j=1}^{N-1} e^{-\alpha_{j} x_{j}^{2}} I_{\theta}\left(\beta x_{j} x_{j-1}\right) x_{j} d x_{j},
$$

where,

$$
\alpha_{j}=\beta\left(1+\frac{\varepsilon^{2}}{2} \omega_{j}\right) ; \quad 0 \leq j \leq N-1 ; \text { and } \beta=\frac{1}{D \varepsilon} .
$$

Now, in order to perform the integrations of Eq.(15), we can use the equality known as Weber formula [22, 23, which is given by 


$$
\int_{0}^{\infty} e^{-\alpha x^{2}} I_{\theta}(a x) I_{\theta}(b x) x d x=\frac{1}{2 \alpha} \exp \left[\frac{a^{2}+b^{2}}{4 \alpha}\right] I_{\theta}\left(\frac{a b}{2 \alpha}\right)
$$

and is valid for $\Re(\theta)>-1, \Re(\alpha)>0$ (here both conditions are fulfilled). The final result is

$$
K\left(x_{b}, t_{b} \mid x_{a}, t_{a}\right)=\sqrt{x_{a} x_{b}} \lim _{N \rightarrow \infty} a_{N} e^{\left(p_{N} x_{a}^{2}+q_{N} x_{b}^{2}\right)} I_{\theta}\left(a_{N} x_{a} x_{b}\right)
$$

where the quantities $a_{N}, p_{N}$ and $q_{N}$ are defined in appendix A. These quantities are related to a function $Q(t)$ that obeys the equation (as usual, we have indicated time derivatives with dots)

$$
\ddot{Q}(t)-\omega(t) Q(t)=0
$$

with the initial condition $Q_{0}=Q\left(t_{a}\right)=0$. In the limit $\varepsilon \rightarrow 0(N \rightarrow \infty)$, we find that (see appendix A)

$$
\begin{gathered}
\lim _{N \rightarrow \infty} a_{N}=\frac{1}{D} \frac{\dot{Q}\left(t_{a}\right)}{Q\left(t_{b}\right)}, \\
\lim _{N \rightarrow \infty} p_{N}=\lim _{\varepsilon \rightarrow 0}\left(\frac{1}{\varepsilon}-\dot{Q}^{2}\left(t_{a}\right) \int_{t_{a}+\varepsilon}^{t_{b}} \frac{d t}{Q(t)^{2}}\right), \\
\lim _{N \rightarrow \infty} q_{N}=-\frac{1}{2 D} \frac{\dot{Q}\left(t_{b}\right)}{Q\left(t_{b}\right)}, .
\end{gathered}
$$

To calculate the second limit, it is necessary to solve Eq. (17). As it was shown in Ref. [18], the complete solution of Eq. (17) can be reduced to quadratures, with the general form given by

$$
Q(t)=k_{1} \mathcal{R}_{t_{a}}(t)+k_{2} \mathcal{S}_{t_{a}}(t)
$$

where

$$
\begin{aligned}
\mathcal{R}_{t_{a}}(t) & =e^{-\int_{t_{a}}^{t} a(s) d s} \\
\mathcal{S}_{t_{a}}(t) & =e^{-\int_{t_{a}}^{t} a(s) d s} \int_{t_{a}}^{t} e^{2 \int_{t_{a}}^{\tau} a(\varsigma) d \varsigma} d \tau .
\end{aligned}
$$


Hence, the solution fulfilling the initial condition $Q_{0}=Q\left(t_{a}\right)=0$ is

$$
Q(t)=\dot{Q}\left(t_{a}\right) \mathcal{S}_{t_{a}}(t)
$$

After replacing this solution into the expressions for $a_{N}, p_{N}$ and $q_{N}$ (see appendix B), we finally arrive to a completely analytical expression for the transition probability

$$
\begin{aligned}
& P\left(x_{b}, t_{b} \mid x_{a}, t_{a}\right)=e^{-\left[\Phi\left(t_{b}\right)-\Phi\left(t_{a}\right)\right]} \frac{\sqrt{x_{a} x_{b}}}{D \mathcal{S}_{t_{a}}\left(t_{b}\right)} \times I_{\theta}\left(\frac{x_{a} x_{b}}{D \mathcal{S}_{t_{a}}\left(t_{b}\right)}\right) \times \\
& \exp \left(\frac{-1}{2 D \mathcal{S}_{t_{a}}\left(t_{b}\right)}\left[\left(\mathcal{R}_{t_{a}}\left(t_{b}\right)+a\left(t_{a}\right)\right) x_{a}^{2}+\left(\frac{1}{\mathcal{R}_{t_{a}}\left(t_{b}\right)}-a\left(t_{b}\right)\right) x_{b}^{2}\right]\right)
\end{aligned}
$$

which can be further simplified as

$$
\begin{gathered}
P\left(x_{b}, t_{b} \mid x_{a}, t_{a}\right)=x_{a}^{\frac{b}{D}+\frac{1}{2}} x_{b}^{-\frac{b}{D}+\frac{1}{2}} \frac{\left[\mathcal{R}_{t_{a}}\left(t_{b}\right)\right]^{\frac{b}{D}-\frac{1}{2}}}{D \mathcal{S}_{t_{a}}\left(t_{b}\right)} \times I_{\theta}\left(\frac{x_{a} x_{b}}{D \mathcal{S}_{t_{a}}\left(t_{b}\right)}\right) \times \\
\exp \left(\frac{-1}{2 D \mathcal{S}_{t_{a}}\left(t_{b}\right)}\left[\mathcal{R}_{t_{a}}\left(t_{b}\right) x_{a}^{2}+\frac{1}{\mathcal{R}_{t_{a}}\left(t_{b}\right)} x_{b}^{2}\right]\right) .
\end{gathered}
$$

It is straightforward to check for some particular choices of $a(t)$ and $b$ that the last expression fulfills the corresponding Fokker-Planck equation. Albeit not so simple, we have also proved it for the general case. The last expression also indicates that, in order to have the explicit form of the propagator we only need to obtain the function $Q(t)$ (the solution of Eq. (17) given in Eq. (21)) for the problem under study (that is, for a given form of the function $a(t)$ ). We will provide a family of solutions for a rather general form of the function $a(t)$ in a subsequent section. Before, we will discuss the possibility of finding a net current at the origin.

\section{A. Flow through the infinite barrier}

Let us first evaluate from Eq. (25) the asymptotic probability distribution, that is

$$
P(x, t)=\lim _{t_{a} \rightarrow-\infty} P\left(x, t \mid x_{a}, t_{a}\right)
$$


In the strongly attractive case (Eq. (3)), it can be easily shown that $\mathcal{S}_{t_{a}}(t)$ diverges and that $\mathcal{R}_{t_{a}}(t)$ goes to zero as $t_{a} \rightarrow-\infty$. Thus, we will make use of the expansion of the modified Bessel function for small argument [22],

$$
I_{\theta}(z)=\frac{1}{\Gamma(\theta+1)}\left(\frac{z}{2}\right)^{\theta}+\mathcal{O}\left(z^{\theta+2}\right) .
$$

Replacing this expansion into Eq. (25) we get,

$$
P(x, t)=\left[\lim _{t_{a} \rightarrow-\infty}\left[x_{a} \mathcal{R}_{t_{a}}(t)\right]^{\frac{b}{D}+\frac{1}{2}+\theta}\right] \frac{2}{\Gamma(\theta+1)} \frac{x^{-\frac{b}{D}+\frac{1}{2}+\theta}}{(2 D g(t))^{1+\theta}} \exp \left(-\frac{x^{2}}{2 D g(t)}\right),
$$

where $g(t)$ is defined as

$$
g(t)=\lim _{t_{a} \rightarrow-\infty} \mathcal{S}_{t_{a}}(t) \mathcal{R}_{t_{a}}(t)
$$

It is clear that unless the condition

$$
1 / 2+b / D+\theta=0
$$

holds, the system cannot reach an asymptotic probability distribution. In fact, the term between the square brackets in Eq. (28) depends on the initial condition. Furthermore, it can be shown that the normalization of Eq. (28) gives a vanishing function of $t$ unless the previous condition holds. Note that Eq. (30) implies, through Eq. (12), $D<-2 b$. This condition gives the maximum value of noise amplitude for the particles to be confined inside the interval $(0, \infty]$.

This encourage us to show explicitly the existence of a noise induced probability current through the infinite barrier at the origin when $D>-2 b$. Before giving a rigorous deduction, let us state a simple argument which provides some clue about the underlying physical mechanism governing this flow. As it is clear from the Langevin equation, the particle is subjected to both deterministic and stochastic forces. If we analyze separately both contributions to the particle movement near the origin, we obtain for the deterministic trajectory $x_{d}(t)=\sqrt{-2 b t}$. Comparing this result with the well known diffusive behavior, where the uncertainty on the particle's position grows as $x_{s}=\sqrt{D t}$, we reobtain the previous condition $D>-2 b$ for the possible appearance of noise induced leakage of particles. 
The probability current at the origin $J\left(x=0, t \mid x_{a}, t_{a}\right)$ can be evaluated from the associated Fokker-Planck equation. In the case $D>-2 b$ we obtain:

$$
J\left(x=0, t \mid x_{a}, t_{a}\right)=\left.\left(-a x-\frac{b}{x}-\frac{D}{2} \frac{\partial}{\partial x}\right) P\left(x, t \mid x_{a}, t_{a}\right)\right|_{x=0}=-D\left(\frac{b}{D}+\frac{1}{2}\right) J_{x_{a}, t_{a}}(t),
$$

where it can be shown that $J_{x_{a}, t_{a}}(t)$ is a positive function of time for any given initial condition. Therefore, we have obtained a non-zero negative current, as previously stated

\section{B. Asymptotic probability distribution}

In the case $D<-2 b$ there is no probability leakage. In fact, the asymptotic probability distribution can be obtained from Eq. (32) and is given by:

$$
P(x, t)=\frac{2}{\Gamma(\theta+1)} \frac{x^{-\frac{2 b}{D}}}{(2 D g(t))^{1+\theta}} \exp \left(-\frac{x^{2}}{2 D g(t)}\right)
$$

which can be easily shown to be normalizable.

It is worth to study how the properties of the elastic parameter function $a(t)$ influences the behavior of the function $g(t)$, which reflects the time evolution of the width of the probability distribution. First, note that from the definition of $g(t)$ given in Eq. (29) we can obtain

$$
\dot{g}(t)=-2 a(t) g(t)+1 \text {. }
$$

From this equation it can be deduced that $g(t)>0 \forall t$, as must be expected for any well behaved probability distribution. In addition, it can be proved that in order to confine the particle in a small region of width $\sqrt{g(t)} \sim \sqrt{\epsilon}$ an attractive force of order $a(t) \sim 1 / \epsilon$ is needed. On the other hand, a small attractive force of order $a(t) \sim \epsilon$, gives a broad

\footnotetext{
${ }^{1}$ This somewhat counter-intuitive flux has an interesting quantum counterpart in the "fall to the center" effect studied by Landau [24].
} 
distribution with $g(t) \sim 1 / \epsilon$. The limiting cases for $P(x, t)$ corresponding to an unbounded spreading $(a(t) \rightarrow 0 \Rightarrow g(t) \rightarrow \infty)$, and to an asymptotically approach to a $\delta(x)$ distribution $(a(t) \rightarrow \infty \Rightarrow g(t) \rightarrow 0)$, can be also obtained.

From the previous paragraph it is clear that even in the strongly attractive situation (see Eq. (3)) the probability distribution may exhibit an unbounded spreading. In fact, we have already shown that even in the monostable situation $a(t)>0$, but where the strength vanishes in time, $g(t)$ grows indefinitely. Therefore, in order to obtain a non-divergent width of the probability distribution, the conditions on the attractive term have to be stronger than the one imposed by Eq. (3). We may infer that the localized-probability condition should be related to a non-vanishing attractive strength of the time averaged potential.

\section{General localization conditions}

Let us discuss the set of conditions that ensures the asymptotic localization of the probability distribution. From the analysis of Eq. (33) it is clear that in order to guarantee a non divergent $g(t)$ the elastic parameter should have the following properties. Its accumulated strength is positive, i. e.

$$
\int_{t_{i}}^{t} a(\tau) d \tau=c>0 \quad \forall t
$$

where $c$ is an arbitrary constant and $t_{i}$ is the nearest time which fulfills the previous equation. This condition is clearly fulfilled if the potential is strongly attractive. We may also infer

that the accumulated attractive effect (Eq. (34)) should be non-vanishing. In other words, the elapsed time where the accumulated strength reaches the given constant $c$ is bounded, that is

$$
t-t_{i}=\Delta t \leq \Delta t_{u} \quad \forall t
$$

where $\Delta t_{u} \equiv \Delta t_{u}(c)$ is the mentioned upper bound for the elapsed time. It is evident that condition (35) is more restrictive than the one imposed by Eq. (3). 
In the following section we will provide a family of examples where the probability is asymptotically localized.

\section{A FAMILY OF ANALYTICAL SOLUTIONS}

As already mentioned, to obtain the final expression for the diffusion propagator, we must first solve Eq. (17) for a given choice of the function $a(t)$. Because the frequency $\omega(t)$ depends only on the harmonic term of the potential, we can make use of any known solution of the simpler harmonic case. A method to generate a whole family of analytical solutions has been proposed in Ref. [18 for the time-dependent harmonic oscillator. In order to reach such a goal the elastic parameter was written in the following form

$$
a(t)=f(t)+\frac{1}{2} \frac{\dot{f}(t)}{f(t)}
$$

This allows us to find the corresponding independent solutions of Eq. (17)

$$
\begin{aligned}
q_{1}(t) & =\frac{\sinh (F(t))}{\sqrt{f(t)}} \\
q_{2}(t) & =\frac{\cosh (F(t))}{\sqrt{f(t)}},
\end{aligned}
$$

where $F(t)=\int_{t_{0}}^{t} f(s) d s$, indicating that $f(t)$ must be an integrable function. The solution which satisfies the initial condition $Q\left(t_{a}\right)=0$ reduces to

$$
Q(t)=\dot{Q}\left(t_{a}\right) \frac{\sinh \left(F(t)-F\left(t_{a}\right)\right)}{\sqrt{f(t) f\left(t_{a}\right)}} .
$$

With this result, the transition probability in Eq. (25) adopts the analytical form

$$
\begin{aligned}
P\left(x_{b}, t_{b} \mid\right. & \left.x_{a}, t_{a}\right)=\mathrm{e}^{\left[\frac{1}{2}-\frac{b}{D}\right]\left(F\left(t_{b}\right)-F\left(t_{a}\right)\right)}\left(\frac{f\left(t_{a}\right)}{f\left(t_{b}\right)}\right)^{\frac{b}{2 D}-\frac{1}{4}} x_{a}^{\frac{b}{D}+\frac{1}{2}} x_{b}^{-\frac{b}{D}+\frac{1}{2}} \frac{\sqrt{f\left(t_{b}\right) f\left(t_{a}\right)}}{D \sinh \left(F\left(t_{b}\right)-F\left(t_{a}\right)\right)} \\
& \times I_{\theta(b)}\left(\frac{x_{a} x_{b}}{D} \frac{\sqrt{f\left(t_{b}\right) f\left(t_{a}\right)}}{\sinh \left(F\left(t_{b}\right)-F\left(t_{a}\right)\right)}\right) \\
& \exp \left(-\frac{f\left(t_{a}\right) e^{-\left(F\left(t_{b}\right)-F\left(t_{a}\right)\right)} x_{a}^{2}+f\left(t_{b}\right) e^{\left(F\left(t_{b}\right)-F\left(t_{a}\right)\right)} x_{b}^{2}}{2 D \sinh \left(F\left(t_{b}\right)-F\left(t_{a}\right)\right)}\right) .
\end{aligned}
$$

Hence, we have obtained a completely analytical expression for the propagator in Eq. (40), that only depends on the choice of the elastic parameter $a(t)$. 


\section{FINAL REMARKS}

In this work we obtained the exact expression for the diffusion propagator in the time dependent anharmonic potential $V(x, t)=\frac{1}{2} a(t) x^{2}+b \ln x$ for a rather general choice of the elastic parameter. The knowledge of the exact form of the propagator can be useful to model different physical and biological phenomena. Particularly interesting problems, suitable to be studied taking advantage of this results, are realistic non-symmetric neuron membrane potentials [25] and the phenomenon of stochastic resonance in a monostable zero-dimensional potential, in spatially extended systems, and in several neuron firing models, among others. A complete and recent review of these stochastic resonance topics can be found in the work of Gammaitoni et al [7]. On the other hand, the knowledge of the exact propagator in the indicated time-dependent anharmonic potential can be useful as a benchmark to test approximate numerical or analytical procedures. Among them we can refer to some of the problems discussed in [26].

Among the several studies of stochastic resonance in monostable systems, it has been shown using scaling arguments and numerical experiments, that the signal to noise ratio is a monotonically increasing function of the noise amplitude [27]. By contrast, it is quite clear that this increase in the response of the system cannot be unbounded. We shall present elsewhere our own results concerning the phenomenon of stochastic resonance in a system described by the potential discussed in the present paper. In this regard, the relation between the maximum noise amplitude and the deterministic force near the origin proved to be a meaningful cutoff for the increase of the response.

It is worth to remark here that the limit $b \rightarrow 0$ is a (kind of) singular one. The naive point of view will be that, in such a limit, the form of the propagator in Eq. (25) shall reduce to

the one corresponding to the case of the harmonic time-dependent potential $V(x) \sim \frac{1}{2} a(t) x^{2}$. However, this limit corresponds to a harmonic time-dependent potential for $x>0$ with an absorbing boundary condition at $x=0$. Then, it should be possible to re-obtain the limit $b \rightarrow 0$ of the diffusion propagator found in this paper $\left(P_{0}\left(x_{b}, t_{b} \mid x_{a}, t_{a}\right)\right)$ from the one 
obtained in Ref. [18] for the harmonic time-dependent case $\left(P_{h}\left(x_{b}, t_{b} \mid x_{a}, t_{a}\right)\right)$ simply as $P_{0}\left(x_{b}, t_{b} \mid x_{a}, t_{a}\right)=P_{h}\left(x_{b}, t_{b} \mid x_{a}, t_{a}\right)-P_{h}\left(-x_{b}, t_{b} \mid x_{a}, t_{a}\right)$. It can be easily proved that this is indeed the case.

\section{ACKNOWLEDGMENTS}

DES and HSW thanks for the kind hospitality extended to them during their stay at the ICTP, Trieste, Italy. DES thanks for support from UBA through a FOMEC grant, Argentina, and HSW for partial support from CONICET, and ANPCyT (Argentinian agencies) and from CEB, Bariloche, Argentina.

\section{APPENDIX: A}

The quantities $a_{N}, p_{N}$ and $q_{N}$ are defined according to

$$
\begin{aligned}
& a_{N}=\beta \prod_{j=1}^{N-1} \frac{\beta}{2 \gamma_{j}} ; \quad \gamma_{1}=\alpha_{1}, \quad \gamma_{j}=\alpha_{j}-\frac{\beta^{2}}{4 \gamma_{j-1}} \\
& p_{N=}-\frac{\beta}{2}+\sum_{j=1}^{N-1} \frac{\beta_{j}^{2}}{4 \gamma_{j}} ; \quad q_{N}=-\frac{\beta}{2}+\frac{\beta^{2}}{4 \gamma_{N-1}} \\
& \beta_{1}=\beta ; \quad \beta_{j}=\beta \prod_{k=1}^{j-1} \frac{\beta}{2 \gamma_{k}}
\end{aligned}
$$

In order to determine the limiting (when $N \rightarrow \infty$ ) values of $a_{N}, p_{N}$, and $q_{N}$, it is usefull to define the following auxiliary quantities

$$
\lambda_{j}=\frac{2}{\beta} \gamma_{j} ; \quad \Lambda_{k}=\prod_{j=1}^{k} \frac{1}{\lambda_{j}},
$$

with $\alpha_{j}$ as defined after Eq. (14) and $\gamma_{j}$ adopting the form

$$
\gamma_{j}=\beta\left(1+\frac{\varepsilon^{2} \omega_{j}}{2}\right)-\frac{\beta^{2}}{4 \gamma_{j-1}},
$$

that allows us to obtain the following equation for $\lambda_{j}$ 


$$
\lambda_{j}=2\left(1+\frac{\varepsilon^{2} \omega_{j}}{2}\right)-\frac{1}{\lambda_{j-1}} .
$$

If we now define that $\lambda_{j}=\frac{Q_{j+1}}{Q_{j}}$, the last equation can be rewritten as

$$
Q_{j+1}-2 Q_{j}+Q_{j-1}=\omega_{j} \varepsilon^{2} Q_{j}
$$

which, in the limit $N \rightarrow \infty$ (and $\varepsilon \rightarrow 0$ ), becomes Eq. (17), with the initial condition $Q_{0}=Q\left(t_{a}\right)=0$, that follows from Eq. (A5).

Finally, we can express the coefficients $a_{N}, p_{N}$, and $q_{N}$ as functions of the new variables

$$
\begin{gathered}
a_{N}=\beta \Lambda_{N-1}=\beta \frac{Q_{1}}{Q_{N}}=\beta \frac{Q_{1}-Q_{0}}{Q_{N}}=\beta \varepsilon\left(\frac{Q_{1}-Q_{0}}{\varepsilon}\right) \frac{1}{Q_{N}} \\
p_{N}=-\frac{\beta}{2}\left(1-\sum_{j=1}^{N-1} \frac{Q_{1}^{2}}{Q_{j+1} Q_{j}}\right) \\
q_{N}=-\frac{\beta \varepsilon}{2}\left(\frac{Q_{N}-Q_{N-1}}{\varepsilon Q_{N}}\right) .
\end{gathered}
$$

\section{APPENDIX: B}

The replacement of the general solution for $Q(t)$ indicated in Eq. (23) into Eqs. $(18, \sqrt[19]{20})$, leads us to obtain the limiting values of $a_{N}, p_{N}$ and $q_{N}$. For $p_{N}$ we find

$$
\lim _{N \rightarrow \infty} p_{N}=\lim _{\varepsilon \rightarrow 0} \frac{-1}{2 D}\left(\frac{1}{\varepsilon}+\frac{1}{\int_{t_{a}}^{t_{b}} d \tau e^{2 \int_{t_{a}}^{\tau} a(\varsigma) d \varsigma}}-\frac{1}{\int_{t_{a}}^{t_{a}+\varepsilon} d \tau e^{2 \int_{t_{a}}^{\tau} a(\varsigma) d \varsigma}}\right) .
$$

Making a Taylor expansion up to second order in $\varepsilon$ of the last denominator we can calculate the limit in Eq. (B1) yielding

$$
\lim _{N \rightarrow \infty} p_{N}=\frac{-1}{2 D}\left(\frac{1}{\int_{t_{a}}^{t_{b}} d \tau e^{2 \int_{t_{a}}^{\tau} a(\varsigma) d \varsigma}}+a\left(t_{a}\right)\right) .
$$

The expressions for $a_{N}$ and $q_{N}$, in terms of the explicit form for $Q(t)$ results in

$$
\begin{gathered}
\lim _{N \rightarrow \infty} q_{N}=\frac{-1}{2 D}\left(\frac{e^{\int_{t_{a}}^{t_{b}} a(\varsigma) d \varsigma}}{\int_{t_{a}}^{t_{b}} d \tau e^{2 \int_{t_{a}}^{\tau} a(\varsigma) d \varsigma}}-a\left(t_{b}\right)\right) \\
\lim _{N \rightarrow \infty} a_{N}=\frac{1}{D} \frac{e^{\int_{t_{a}}^{t_{b}} a(\varsigma) d \varsigma}}{\int_{t_{a}}^{t_{b}} d \tau e^{2 \int_{t_{a}}^{\tau} a(\varsigma) d \varsigma}} .
\end{gathered}
$$




\section{REFERENCES}

[1] N. G. van Kampen, Stochastic Process in Physics and Chemistry, (North-Holland, New York 1981); C. W. Gardiner, Handbook of Stochastic Methods (Springer-Verlag, New York, 1985); H. S. Wio, An Introduction to Stochastic Processes and Nonequilibrium Statistical Physics (World Scientific, Singapore, 1994).

[2] G. Nicolis and I. Prigogine, Self-Organization in Non-Equilibrium Systems (Wiley, New York, 1977).

[3] D. Walgraef, Spatio-temporal pattern formation, (Springer-Verlag, New York, 1997).

[4] W. Horsthemke and R. Lefever, Noise induced transitions, (Springer-Verlag, New York, 1986).

[5] C. van den Broeck, J. M. R. Parrondo and R. Toral, Phys. Rev. Lett. 73, 3395 (1994);

C. van den Broeck, J. M. R. Parrondo, R. Toral and R. Kawai, Phys. Rev. E 55, 4084 (1995); S. Mangioni, R. Deza, H. S. Wio and R. Toral, Phys. Rev. Lett. 79, 2389 (1997); S. Mangioni, R. Deza, H. S. Wio and R. Toral, submitted to Phys. Rev. E (1998).

[6] R. J. Deissler, J. Stat. Phys. 54, 1459 (1989); K. L. Babcock, G. Ahlers and D. S. Cannell, Phys. Rev. E 50, 3670 (1994); M. Neufeld, D. Walgraef and M. San Miguel, Phys. Rev. E 54, 6344 (1996).

[7] F. Moss, in Some Problems in Statistical Physics, edited by G. Weiss (SIAM, Philadelphia, 1992); Proceedings of the 2nd International Workshop on Fluctuations in Physics and Biology: Stochastic Resonance, Signal Processing and Related Phenomena, edited by A. Bulsara et al., Nuovo Cim. D 17 nro.7-8 (1995); L. Gammaitoni, P. Hänggi, P. Jung and F. Marchesoni, Rev. Mod. Phys., 70, 223 (1998).

[8] J. F. Lindner, et al., Phys. Rev. Lett. 75, 3 (1995) and Phys. Rev. E 53, 2081 (1996); H. S. Wio, Phys. Rev. E 54, R3075 (1996); F. Marchesoni, L. Gammaitoni and A. Bulsara, Phys. Rev. Lett. 76, 2609 (1996); F. Castelpoggi and H. S. Wio, Europhys. Lett. 38, 
91 (1997); F. Castelpoggi and H. S. Wio, Phys. Rev. E 57, 5112 (1998); M. Kuperman, H.S. Wio, G. Izús and R. Deza, Phys. Rev. E 57, 5122 (1998); M. Kuperman, H.S. Wio, G. Izús, R. Deza and F. Castelpoggi, Physica A 257, 275 (1998).

[9] N. Wiener, J. Math. 2, 131 (1923); Proc. London Math. Soc. 22, 454 (1924); Acta Math. 55, 117 (1930).

[10] R. P. Feynman, Rev. Mod. Phys. 20, 367 (1948).

[11] L. Onsager and S. Machlup, Phys. Rev. 91, 1505 (1953); 91, 1512 (1953).

[12] L. Pesquera, M. A. Rodriguez and E. Santos, Phys. Lett. A 94, 287 (1983); R. Graham, Weak Noise Limit and Nonequilibrium Potentials of Dissipative Dynamical Systems, in Instabilities and Nonequilibrium Structures, Eds. E. Tirapegui and D. Villaroel (D. Reidel, Dordrecht, 1987); J. F. Luciani and A. Verga, J. Stat. Phys. 50, 567 (1988); A. Forster and A. S. Mikhailov, Phys. Lett. A 126, 459 (1988); H. S. Wio, P. Colet, M. San Miguel, L. Pesquera and M. A. Rodriguez, Phys. Rev. A 40, 7312 (1989); P. Hänggi; Z. Phys. B 75, 275 (1989); H. S. Wio and D. H. Zanette; Phys. Rev. E 47, 384 (1993); H. S. Wio, C. Budde, C. Briozzo, P. Colet, Int. J. Mod. Phys. B 9, 679 (1995); F. Castro, H. S. Wio and G. Abramson, Phys. Rev. E 52, 159 (1995); F. Castro, A. Sanchez and H. S. Wio, Phys. Rev. Lett. 75, 1691 (1995); M. Dykman and K. Lindenberg; Fluctuations in Nonlinear Systems Driven by Colored Noise, in Some Problems in Statistical Physics, Ed. G. Weiss (SIAM, Philadelphia, 1993).

[13] Some of the several books dealing with the path integral approach to quantum mechanics are:

(a) R. P. Feynman and A. R. Hibbs, Quantum mechanics and path integrals (McGrawHill, New York, 1965); (b) L. S. Schulman, Techniques and applications of path integration (Wiley, New York, 1981); (c) B. Felsager, Geometry, Particles and Fields (Odense University Press, Odense, 1985), chp.5; (d) V. Sa-yakanit et al., Eds, Path Integrals from meV to MeV, (World Scientific, Singapore, 1989); (e) H. Kleinert, Path integrals in 
quantum mechanics, statistics and polymer physics (World Scientific, Singapore, 1990); (f) H. Cerdeira, et al, Eds, Path Integration: Trieste 1991, (World Scientific, Singapore, 1992); (g) D. C. Khandekar, S. W. Lawande, Path integral methods and their applications (World Scientific, Singapore, 1993); (h) A. Das, Field theory: a path integral approach (World Scientific, Singapore, 1994);

Besides these books, there a few other that describe path integral techniques mainly in relation with diffusion and stochastic processes:

(i) F. Langouche, D. Roekaerts, and E. Tirapegui, Functional Integration and Semiclasical expansions (Reidel, Dordrecht, 1982); (j) M. Freidlin, Functional integration and partial differential equations, (Princenton Univ. Press, 1985); (k) F. W. Wiegel, Introduction to path integral methods in physics and polymer science (World Scientific, Singapore, 1986); (1) H. S. Wio, Introducción a las integrales de camino (Universitat de les Illes Balears, Palma de Mallorca, 1990).

[14] P.C. Martin, E.D. Siggia and H.A. Rose, Phys. Rev. A 8, 423 (1973).

[15] H.K. Jensen, Z. Phys. B 23, 377 (1976); C. De Dominicis, J. Phys. (Paris) Colloque C1, 37, 247 (1976); C. De Dominicis and L. Peliti, Phys. Rev. B 18, 353 (1978).

[16] I. H. Duru and H. Kleinert, Phys. Lett. B 84, 30 (1979), and Fortschr. Physik 30, 401 (1982); R. Ho and A. Inomata, Phys. Rev. Lett. 48, 231 (1982); A. Inomata, Phys. Lett. A 87, 387 (1983); N. Pak and I. Sokman, Phys. Rev. A 30, 1629 (1984); I. H. Duru, Phys. Rev. D 28, 2689 (1983); N. Pak and I. Sokman, Phys. Lett. A 100, 327 (1984); A. Inomata, Phys. Lett. A 101, 253 (1984); H. Kleinert, Mod. Phys. Lett. A 4, 2329 (1989); Phys. Lett. B 224, 313 (1989) and Phys. Lett. B 236, 315 (1990).

[17] Ph. Blanchard and M. Siruge, J. Math. Phys. 22, 1372 (1981); A. Young and C. DeWittMorette, Ann. Phys. (N.Y.) 169, 140 (1984).

[18] C. D. Batista, G. Drazer, D. Reidel and H. S. Wio, Phys. Rev. E 54, 86 (1996). 
[19] A. Pelster and H. Kleinert, Phys. Rev. Lett. 78, 565 (1997).

[20] N. G. Stocks, N. D. Stein and P. V. E. McClintock, J. Phys. A 26, L385 (1993); M. J. Dykman, D. G. Luchinsky, R. Manella, P. V. E. McClintock, N. D. Stein and N. G. Stocks, J. Stat. Phys. 70, 479 (1993); A. N. Grigorenko, S. I. Nikitin and G. V. Roschepkin, Phys. Rev. E 56, R4907 (1997).

[21] D. C. Khandekar and S. V. Lawande; J. Math. Phys. 16, 384 (1975).

[22] G. N. Watson, A treatise in the theory of Bessel functions, (Cambridge University Press, Cambridge, England, 1962).

[23] D. Peak and A. Inomata; J. Math. Phys. 10, 1422 (1969).

[24] L. D. Landau and E. M. Lifshitz, Quantum Mechanics (Pergamon, New York, 1958) Sec. 35 .

[25] This is the subject of a work in preparation.

[26] L. Schimansky-Geier and T. Pöschel, editors, Stochastic Dynamics (Springer-Verlag, Berlin, 1997); U. Deininghaus and R. Graham, Z.Phys. B 34, 211 (1979); P. Hänggi, Chem. Phys. 180157 (1994).

[27] J. M. Vilar and J. M. Rubi, Phys. Rev. Lett. 77, 2863 (1996). 